\title{
Market Organisation and Trading Relationships
}

\author{
GÉrard Weisbuch ${ }^{(1)}$
}

Alan Kirman ${ }^{(2)}$

and Dorothea Herreiner ${ }^{(3)}$

(1) Laboratoire de Physique Statistique

de l'Ecole Normale Supérieure,

24 rue Lhomond, F 75231 Paris Cedex 5, France.

(2) GREQAM

EHESS et Université d'Aix-Marseille III

13002 Marseille, France

(3) Economic Theory III

University of Bonn, Adenauerallee 24 - 26

53113 Bonn, Germany

email:weisbuch@physique.ens.fr

March 3, 2000

Keywords: market organisation, reinforcement learning, customer loyalty, perishable goods.

\section{Introduction}

Many markets are characterised by trading relationships. Individuals systematically trade with particular partners in certain markets whilst in others no such stable links are observed. Some other markets exhibit a mixture of stable links and "searching" behaviour. Yet the way in which such organisation develops and its economic consequences are not considered in most standard theoretical models. In a Walrasian equilibrium, for example, the following questions are left unanswered: 
- How do agents get the information about who demands or supplies which good at what price? Who determines those prices?

- How is that information used to determine who will make which transaction with whom, thereby clearing the market at each stage and determining market organisation in the long run?

One of the objectives of this paper is to examine a situation in which individuals set prices and the way in which those who wish to buy, at those prices, become matched with those who wish to sell. In standard search models (see e.g. Diamond (1989)) ${ }^{1}$, buyers sample sellers according to some rule and buy from the cheapest. All sellers are anonymous and are searched with equal probability. There is no memory of where favourable opportunities were found in the past. Such models seem to be plausible for transactions which take place infrequently. Yet many markets are ones on which individuals trade frequently with each other. This particularly true for markets for perishable goods. Furthermore, in the latter case, since sellers cannot hold inventories, they only supply the quantities they expect to sell during one session. The essential risk, in our stylised context, for a buyer is not that of paying too high a price but rather of not being served at all. Hence, rather than gathering information about different sellers at each session, a buyer may find it worthwhile to use the experience gained from transactions with sellers during previous sessions.

In our model, trading relationships develop precisely because buyers learn, in this way, about the value of trading with particular partners. Such stable trading relationships are also profitable to sellers who can then predict with some accuracy the demand they will face in each session and determine their supply accordingly. The more loyal the customers, the better the prediction and the more likely the customer is to find the goods he is seeking. Thus the establishment of regular trading relationships may be mutually profitable. The basic aim of this paper is to examine the extent to which agents in a simple model of such markets will learn their way to establishing trading links and to characterize the conditions under which this happens.

We emphasize that the intrinsic uncertainty in our model is due to the behaviour of the buyers. There is no exogenous uncertainty about the parameters of which agents learn. Thus our model differs from those of (Felli and Harris (1996), Bergemann and Valimaki (1994) and (1996)), in which agents learn about some exogenous random variable and strategic behaviour influences such learning. Our model, on the other hand, uses a simple rule of thumb for learning with no strategic thinking. This would seem to be well adapted to a situation with no a priori stable distribution of the quantities available at each shop. This the case for buyers in the Marseille wholesale fish market from which our empirical evidence is taken.

In this paper, we limit our attention to situations in which individuals have to rely on their own experience and do not observe that of others directly. We shall be interested here, in particular, in markets in which transactions are not made public, and no prices are posted. In such markets agents have to rely on their own information. We will therefore develop a model which seeks to explain some of the

\footnotetext{
${ }^{1}$ for more elaborate models, see e.g. Fisher (1973) and Lesourne (1992).
} 
phenomena that characterise this particular type of market in which learning is from individual past experience. Our emphasis is on the buyers side of the market but the conclusions that we draw will be shown to hold even when different sellers behaviour is introduced. We will adopt an approach which allows us to obtain analytical results for the simplest version of our model and we then use simulations to check that these results still hold in more complicated and realistic versions.

The structure of the paper is as follows. We start by proposing a very simple model of a market for a perishable good, in which at each time step buyers (retailers) meet sellers (wholesalers) and buy quantities of the homogeneous good to resell on their own local market. They do this in a shop which is chosen according to the information gathered during previous purchases. This model is solved analytically using the "mean field" approximation. The theory predicts that two distinct types of behaviour for the agents should be observed according to their learning and choice parameters: some agents should remain loyal to one selected shop, while others should keep on shopping around for ever. We then use multi-agent simulations to study more complex, and more realistic versions of the model, allowing for instance several purchases per buyer during the same day, varying prices, and more complicated adaptive behaviour of buyers and sellers. Our simulations show that the same patterns of dynamic behaviour persist. Finally, we verify that the theoretical predictions which are specific to our model are consistent with empirical data from the wholesale fish market in Marseille.

\section{The Simplest Model}

Let us consider a set of $n$ buyers $i$ and a set of $m$ sellers $j$.

\subsection{Basic Assumptions}

In order to simplify assumptions as much as possible, let us suppose that:

- Customers choose one shop every day according to their memory of previous transactions. As long as the shop has supply, a customer purchases a quantity $q_{i}(t)$ implying a profit $\pi_{i}(t)$. Whether the customer is served when he visits the shop depends on which shop $j$ is visited at time $t$, how many people bought from that shop before, and how much endowment the shop had at the beginning of the day ${ }^{2}$.

- Since the good is perishable and therefore cannot be stored between days, each day a seller supplies a quantity $Q_{j}(t)$ which he expects to sell on that day. In the simplest version of the model, this quantity is simply the quantity he sold yesterday.

- Every day the same market scenario is repeated.

\footnotetext{
${ }^{2}$ In the simplest model, customers visit only only one shop and thus have only one chance to get served everyday.
} 
These simplistic assumptions will be used in sections 2, 3 and 4 . More realistic assumptions will be made in section 5 .

\subsection{Preference coefficients, Learning and Choice Probabili- ties}

Our model seeks to explain trading relationships. Therefore, our assumptions about how buyers choose which shop to visit are crucial. These assumptions ${ }^{3}$ are kept constant throughout the entire paper, i.e. they are the same for the basic model and its extensions.

A buyer has to choose one shop each day. The basic assumption of the present model is that his present choice of which shop to visit is based on his previous experience. His decision rule is therefore a mapping from the time series of the transactions he has had with different sellers and the profits associated with the transactions ${ }^{4}$, $\mathbf{I}(t)$, to the unit simplex $\Delta_{m}$, where $m$ is the number of sellers:

$$
P(t): \mathbf{I}(t) \longrightarrow \Delta_{m}
$$

A point in the simplex $\Delta_{m}$ represents the probabilities with which an individual chooses each of the $m$ sellers.

The mapping $P(t)$ can be decomposed into two components, a mapping from $\mathbf{I}(t)$ to a vector $\mathbf{J}(t)$ of "preference coefficients" and a second mapping from $\mathbf{J}(t)$ to $\Delta_{m}$. The first mapping is an encoding based on a learning process and the second mapping describes the probabilistic choice process.

Let us first specify the learning process of the buyers. By assumption the only information available comes from past transactions, so each buyer has a record for each shop. The profits buyer $i$ made when buying from shop $j$ are mapped into the preference coefficient $J_{i j}$ by adding profits every period and discounting previous profits at a constant rate $\gamma$. Since we use discrete time for transactions, preferences are updated at each time step according to:

$$
J_{i j}(t)=(1-\gamma) \cdot J_{i j}(t-1)+\pi_{i j}(t), \quad \forall i, \forall j
$$

In other words, at each time step, all preference coefficients are discounted at a constant rate, and the preference coefficient for the shop with which a transaction occurs is increased by the profit made in that shop. Preference coefficients thus appear as the sum of discounted past profits. Discounting can be interpreted in different ways: it describes gradual forgetting of past events; it also serves to ensure that information is relevant to the current situation. In real life shops do not necessarily have stationary characteristics in terms of the profits that they offer, because of possible changes in

\footnotetext{
${ }^{3}$ The learning and probabilistic choice process described in this section was inspired by the formal neural networks approach to reinforcement learning as described for instance in Weisbuch (1990).

${ }^{4}$ In wholesale markets of perishable goods, profits are more pertinent to retailers than prices since retailers are facing uncertainties related to prices and available quantities. The relation between profits, prices and available quantities are discussed in section 5 .
} 
prices for many possible reasons, in their initial endowment and in their number of customers.

The decision rule used by the buyers then maps these preference coefficients into the choice of a shop. One deterministic way to do so is to choose the shop with the best record, that is the shop with the highest $J_{i j}(t)$. This would amount to mapping the $\mathbf{J}(t)$ into one of the apices of $\Delta_{m}$. However, by doing this, the buyer would become a captive of the selected shop which would then be in a position to diminish the buyer's profit and to increase its own profit by changing prices. The shop could do this until the buyer's profit becomes negative before running any risk of losing that buyer. It is therefore in the buyer's interest to search from time to time among other sellers to check whether he could get a better profit elsewhere. In other words, a reasonable rule-of-thumb for the buyers would be a balance between the deterministic choice in favor of those shops which gave the best profits in the past and random search among other sellers. This raises the well known issue of the trade-off between exploitation of old knowledge and exploration to acquire new knowledge.

We use a probabilistic choice rule here, which characterizes this trade-off with a single parameter $\beta$. We suppose that the decision rule by which a buyer $i$ assigns a probability $P_{i j}$ of visiting seller $j$ is proportional to the exponential of the preference coefficient for that seller. That is:

$$
P_{i j}=\frac{\exp \left(\beta J_{i j}\right)}{\sum_{j^{\prime}} \exp \left(\beta J_{i j^{\prime}}\right)}, \quad \forall i, \forall j
$$

where $\beta$, the discrimination rate, measures the non-linearity of the relationship between the probability $P_{i j}$ and the preference coefficient $J_{i j}$. This specification ${ }^{5}$ allows for any choice rule in the range of equal probabilities $(\beta=0)$ to best-reply $(\beta=\infty)$.

In our case, the exponential rule can be derived directly (see Brock (1993) for a discussion). This is done by maximizing the weighted sum $F_{i}$ of two terms; one of which favors immediate profit:

$$
G_{i}=\sum_{j} P_{i j} J_{i j}
$$

$G_{i}$ is approximately the expected discounted sum of profits. The other term favors search. To maximise the information gained during visits, buyers should maximize the Shannon entropy ${ }^{6}$ of the distribution of search probabilities:

$$
S_{i}=-\sum_{j} P_{i j} \log P_{i j}
$$

The function $F_{i}$ to be maximized is then a linear combination of preferences and entropy terms:

$$
F_{i}=\beta G_{i}+S_{i}
$$

\footnotetext{
${ }^{5}$ The exponential rule has been widely used in economics and elsewhere. Several justifications for its use are given in the discrete choice literature, see e.g. Anderson et al. (1992).

${ }^{6}$ Entropy is a measure of the disorder of a system; it is maximal (for each $i$ ) if all $P_{i j}=1 / \mathrm{m}$, "the most random probability measure" as Brock (1993) calls it. Entropy is minimized if $P_{i j}=1$ for one $j$ and the other $P_{i j}=0$.
} 
The smaller $\beta$ the stronger the weight given to disorder, i.e. to information gathering at different shops. The larger $\beta$ the more important (short-run) payoff concerns. Setting the derivatives of $F_{i}$ with respect to $P_{i j}$ equal to zero under the constraint that the sum of the probabilities is 1 gives equation (3).

\section{Mean Field Approach}

The simple model can be formally analysed within the framework of the Mean Field approach. This consists in replacing randomly fluctuating quantities by their average, thus neglecting fluctuations. It is only an approximation, but is often convenient to obtain at least a qualitative understanding of the behavior of the system. ${ }^{7}$

The model is soluble in the continuous limit, when the changes of variables are small at each time step, i.e. $\gamma \rightarrow 0$. Equation (2) can be expressed as a difference equation in $\tau$ by multiplying $\gamma$ and $\pi_{i j}(t)$ by $\tau$ and then rewriting it as:

$$
\frac{J_{i j}(t+\tau)-J_{i j}(t)}{\tau}=-\gamma J_{i j}(t)+\pi_{i j}(t)
$$

Taking the limit for $\tau \rightarrow 0$, leads to a stochastic differential equation

$$
\frac{d J_{i j}}{d t}=-\gamma J_{i j}+\pi_{i j}
$$

in $\pi_{i j}$. The Mean Field approximation consists in replacing the $\pi_{i j}$ by its expected value $\left\langle\pi_{i j}\right\rangle$, thereby transforming the stochastic differential equation into a deterministic differential equation.

The time evolution of $J_{i j}$ is thus approximated by the following equations:

$$
\begin{gathered}
\frac{d J_{i j}}{d t}=-\gamma J_{i j}+<\pi_{i j}> \\
<\pi_{i j}>=\operatorname{Prob}\left(q_{i}>0\right) \cdot \pi_{i j} \frac{\exp \left(\beta J_{i j}\right)}{\sum_{j^{\prime}} \exp \left(\beta J_{i j^{\prime}}\right)} ;
\end{gathered}
$$

the fraction represents the probability that buyer $i$ visits shops $j$; $\operatorname{Prob}\left(q_{i}>0\right)$ is the probability that the shop still has goods to sell when the buyer comes to shop $j$, in which case he gets a quantity $q_{i}$ resulting in profit $\pi_{i j}$. Suppose the market converges to a stationary state in which buyers' preference coefficients do not change. Such a state is called an equilibrium in dynamical systems theory and it is obtained by setting the derivatives (equation (7)) equal to zero.

\footnotetext{
${ }^{7}$ The limits of applicability of the Mean Field Theory are an advanced topic in Statistical Physics discussed for instance in Brout (1965). Some ideas are discussed in an economics context in Aoki (1996). Even without a full discussion (well beyond the scope of the present paper), we might expect that the Mean Field Theory applies reasonably well to the present case with no a priori connection structure. Anyway, even in those cases where its predictions are quantitatively inaccurate, the Mean Field Theory is still good enough to predict the transitions and some dynamical properties in their neighbourhood. This is sufficient for our purpose of solving a simple model to gain some insight in the structure of the market and to calibrate the simulation results.
} 
Let us consider the simplest case of two shops and to further simplify computation, let us suppose, for the moment being, that $\operatorname{Prob}\left(q_{i}>0\right)=1$, which happens when buyers always find what they require at the shop they visit. (If this were always the case, there would be no rationale for the learning and choice algorithm described in section 2. We take here $\operatorname{Prob}\left(q_{i}>0\right)=1$ only as a limiting case allowing analytical computations which are then checked against numerical simulations in section 4 with $\left.\operatorname{Prob}\left(q_{i}>0\right) \leq 1\right)$.

\subsection{The order/disorder transition for the symmetric case}

To make computations easier, let us first suppose for the moment that profits in both shops are equal to $\pi$ (see the next section for unequal profits). The equilibrium relations are in this case:

$$
\begin{aligned}
\gamma J_{1} & =\pi \frac{\exp \left(\beta J_{1}\right)}{\exp \left(\beta J_{1}\right)+\exp \left(\beta J_{2}\right)}, \\
\gamma J_{2} & =\pi \frac{\exp \left(\beta J_{2}\right)}{\exp \left(\beta J_{1}\right)+\exp \left(\beta J_{2}\right)} .
\end{aligned}
$$

We dropped the index $i$ referring to the buyer, and the remaining indices 1 and 2 refer to the sellers. Subtracting equation 10 from equation 9, we see that the difference between the two preference coefficients, $\Delta=J_{1}-J_{2}$, obeys the following implicit equation:

$$
\frac{\gamma \Delta}{\pi}=\frac{\exp (\beta \Delta)-1}{\exp (\beta \Delta)+1}
$$

The right hand side of the equation is the hyperbolic tangent of $\beta \Delta / 2$. The above equation has either one or three solutions according to the slope of the hyperbolic tangent at the origin. If ${ }^{8}$

$$
\beta<\beta_{c}=\frac{2 \gamma}{\pi}
$$

there is only one stable solution $\Delta=0$ and $J_{1}=J_{2}=\frac{\pi}{2 \gamma}$. The average $J_{j}$ are small and equal. A buyer visits both shops approximately half the time, switching at random between the shops. We call such a regime disordered or disorganized.

In the opposite situation, if $\beta>\beta_{c}$, the zero solution is unstable and the other two solutions are stable and symmetric, with one preference coefficient large and the other one small ${ }^{9}$. At the stable solutions a buyer visits one shop with high probability and frequency (high preference coefficient) and the other shop with very low probability and therefore rarely (low preference coefficient). We call such a regime ordered or organized; buyers are loyal.

The transition from the disordered to the ordered regime is abrupt; the difference between the preference coefficients $\Delta$ stays 0 for $\beta<\beta_{c}$, it changes with infinite slope

\footnotetext{
${ }^{8}$ By developing the hyperbolic tangent in series for small values of $\beta \Delta / 2$. See appendix $\mathrm{A}$ for more details.

${ }^{9}$ The ratio between the two preference coefficients is exponential in $\beta \pi / \gamma$; see appendix A.
} 
at $\beta=\beta_{c}$, and it increases approximately by the square root of the distance $\left(\beta-\beta_{c}\right)$ (close to $\Delta=0)$ :

$$
\Delta=\sqrt{\frac{12\left(\beta-\beta_{c}\right)}{\beta^{3}}}
$$

as can be seen in figure 1 obtained by solving equation 11 .

$\mathrm{J}$

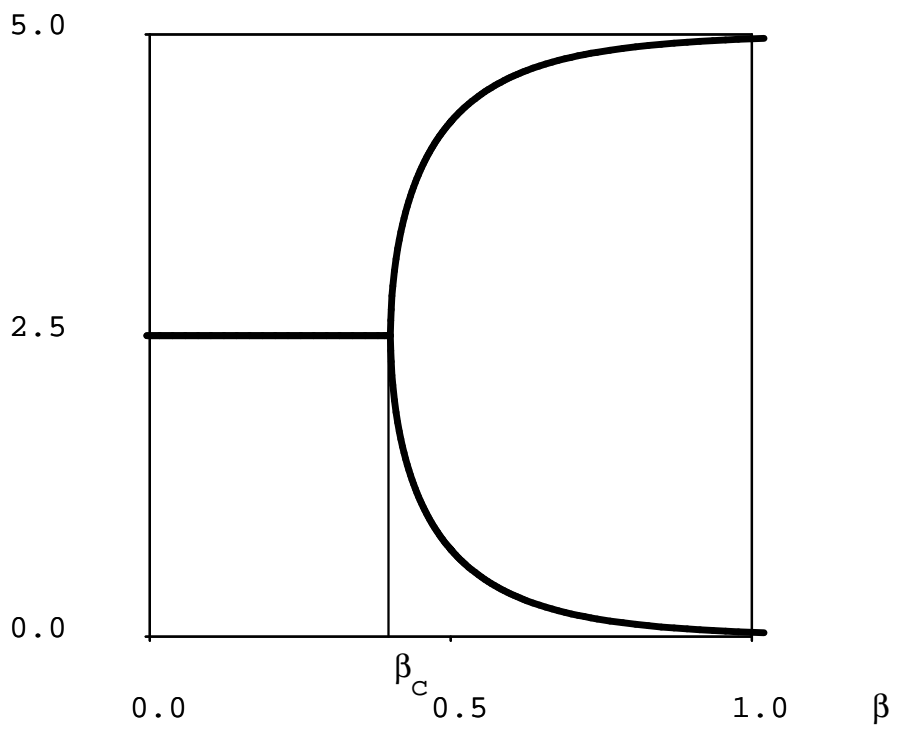

Figure 1: The order/disorder transition in $\beta$. Plot of both equilibrium preference coefficients versus the discrimination rate $\beta$. Below the transition rate $\beta_{c}$, preference coefficients are equal, but they rise or plummet sharply when the discrimination rate $\beta$ increases above the transition. When profits in both shops are equal (as in this figure), either loyalty describes the upper branch, while the other describes the lower branch. (The figure is drawn for two shops with $\pi=1$ and $\gamma=0.2$ using GRIND software, De Boer 1983).

In the case of $m$ shops, the fixed point equations are:

$$
J_{j}=\frac{\pi}{\gamma} \frac{\exp \left(\beta J_{j}\right)}{\sum_{k} \exp \left(\beta J_{k}\right)} .
$$

Summing over $j$ the fixed point equations (14) one sees that any solution $\mathbf{J}$ satisfies

$$
\sum_{j} J_{j}=\frac{\pi}{\gamma}
$$


Obviously, the symmetric fixed point

$$
J_{j}=\frac{\pi}{m \gamma} \quad j=1, \ldots, N
$$

satisfies equation (14). The symmetric fixed point is an attractor if and only if the right hand side of equation (14) has a slope smaller than one. This condition is easily checked since the derivative of the denominator of the HRS of equation (14) is zero at the symmetric point, due to equation (15) and equality of the derivatives with respect to the $J_{k}$. We thus obtain:

$$
\beta_{c}=\frac{m \gamma}{\pi}
$$

In this case, there is either one stable stationary point (if $\beta<\beta_{c}$ ), where the customer visits all shops with equal likelihood, or there are $m$ stable stationary points (if $\beta>\beta_{c}$ ), where a buyer is loyal to one of the $m$ shops $^{10}$.

The above analysis shows that as long as the mean field approximation remains valid, the qualitative behavior of the dynamics, ordered or disordered, only depends on the ratio between $\beta$ and $\beta_{c}$. As long as $\beta / \beta_{c}$ is kept constant, changing the original parameters $m, \beta$, and $\pi$, only changes the scale of equilibrium variables such as actual profits of the buyers or the fraction of unsold endowments. The time scale of learning depends on $\gamma$ : order, when achieved, is reached faster for larger values of $\gamma$.

Within the approximations made in this section, buyer dynamics are uncoupled: each buyer behaves independently of other buyers. As a result, if we now consider a set of buyers with a distribution of $\pi, \beta$ and $\gamma$ parameters, we expect to observe two distinct classes of buyers within the same market: loyal buyers with $\beta>\beta_{c}$, who visit the same shop most of the time, and searchers with $\beta<\beta_{c}$, who wander from shop to shop. Indeed, precisely this sort of "division of labour" is observed on the Marseille fish market which was the empirical starting point for this paper and which will be discussed in section 6. Furthermore, because of the sharp transition in behavior when $\beta$ goes across the transition, the distribution of behavior is expected to be bimodal even if the distribution of the characteristics $\pi, \beta$ and $\gamma$ is unimodal.

We can now compare the predictions of our model where agents learn individually from their past experience with those of models where agents imitate each others' behavior through social interactions (Föllmer (1974), Arthur/Lane (1993), Brock/Durlauf (1995), Orléan (1995)). Both types of models exhibit an abrupt phase transition between order for large $\beta$ values and disorder for small $\beta$ values. Two main differences exist.

- In the ordered regime, in the case of imitation, all agents make the same choice (at least when interactions among all agents are a priori possible ${ }^{11}$ ); in our

\footnotetext{
${ }^{10}$ In total there are $2^{m}-1$ equilibria for the differential equations associated with equation (17), however, $2^{m}-1-m$ of these equilibria are not stable.

${ }^{11}$ Imitation favors uniformity, but according to whether one uses a mean field approach (all interactions being possible) as in Arthur/Lane (1993), Brock/Durlauf (1995), Orléan (1995), or Markov random fields (interactions restricted to some neighborhood) as in Föllmer (1974), one observes global or local order. All agents make the same choice in the first case. Different choices can be made in the second case, with local patches of agents making the same choice.
} 
model different agents are loyal to different shops. Imitation and positive social interactions favor uniformity, while decisions based on agents' memory favor diversity.

- In our model heterogeneity of buyer parameters results in having two classes of behavior, searchers and loyal buyers. Order is a property of buyers, not of the market. In imitation models, the market as a whole is organised or disorganised, even in the presence of heterogeneity of agents ${ }^{12}$.

\subsection{Shops offering different profits and hysteresis}

Up to this point we have considered a situation in which sellers propose the same prices, resulting in equal profits for buyers and we now we have to check whether similar results apply when profits differ. Let us come back once more to the simple case of two shops 1 and 2, and now suppose that they offer different prices and hence different profits $\pi_{1}$ and $\pi_{2}$. Replacing profit $\pi$ in equations 9 and 10 by respectively $\pi_{1}$ and $\pi_{2}$, equation 11 becomes:

$$
\frac{\gamma \Delta}{\left(\pi_{1}+\pi_{2}\right) / 2}-\frac{\pi_{1}-\pi_{2}}{\pi_{1}+\pi_{2}}=\frac{\exp (\beta \Delta)-1}{\exp (\beta \Delta)+1}
$$

The equation in $\Delta$ has once more either one or three solutions, depending now on the value of two reduced parameters, the relative profit difference $r=\frac{\pi_{1}-\pi_{2}}{\pi_{1}+\pi_{2}}$ and the ratio between $\beta$ and $\beta_{c}=\frac{4 \gamma}{\left(\pi_{1}+\pi_{2}\right)}$. The geometric interpretation of equation 18 in $\Delta$ is still the intersection of a straight line (the left hand-side of equation 18) with the hyperbolic tangent (its right hand-side). Equation (18) amounts to shifting the left-hand side of equation (11) by $\left(\pi_{1}-\pi_{2}\right) /\left(\pi_{1}+\pi_{2}\right)$ and by replacing the uniform profit by its average on the two shops.

If $\beta$ is above ${ }^{13} \beta_{c}$, the three intersections remain as long as the difference in relative profits $r$ is not too large. The frontier in the two regimes, three versus one solution, in the $\beta, r$ parameter space is described by inequality:

$$
\left(\frac{3 r}{2}\right)^{2}<\left(1-\frac{\beta_{c}}{\beta}\right)^{3}
$$

in the neighborhood of $\beta=\beta_{c}{ }^{14}$.

\footnotetext{
${ }^{12}$ Once more, this statement applies rigorously to the mean field approach. In the case of large heterogeneity of local interactions in Markov random fields, ordered and disordered regions might coexist.

${ }^{13}$ If $\beta<\beta_{c}$ then there remains only one stable solution, in which there is a small difference in preferences proportional to the difference in profits (if $\beta \Delta$ is small):

$$
J_{1}-J_{2} \simeq \frac{4\left(\pi_{1}-\pi_{2}\right)}{\left(\beta_{c}-\beta\right)\left(\pi_{1}+\pi_{2}\right)} .
$$

Compare with footnote 9 .

${ }^{14}$ expression obtained by developing the hyperbolic tangent in series for small values of $\beta \Delta / 2$.
} 
As we could have easily guessed, the existence of two regimes separated by an abrupt phase transition does not depend upon the simplifying assumption of symmetry between the two shops. The above analysis can be generalised to a larger number of shops and exhibit transitions in the number of solutions with respect to relative profit differences among shops and average profit.

In the organised regime, which of the two asymmetric intersections is actually reached by the learning dynamics depends on initial conditions. The following analysis of the hysteresis effect can give us some clues about the consequences of changes in the profit offered by the different sellers ${ }^{15}$.

$\mathrm{J}$

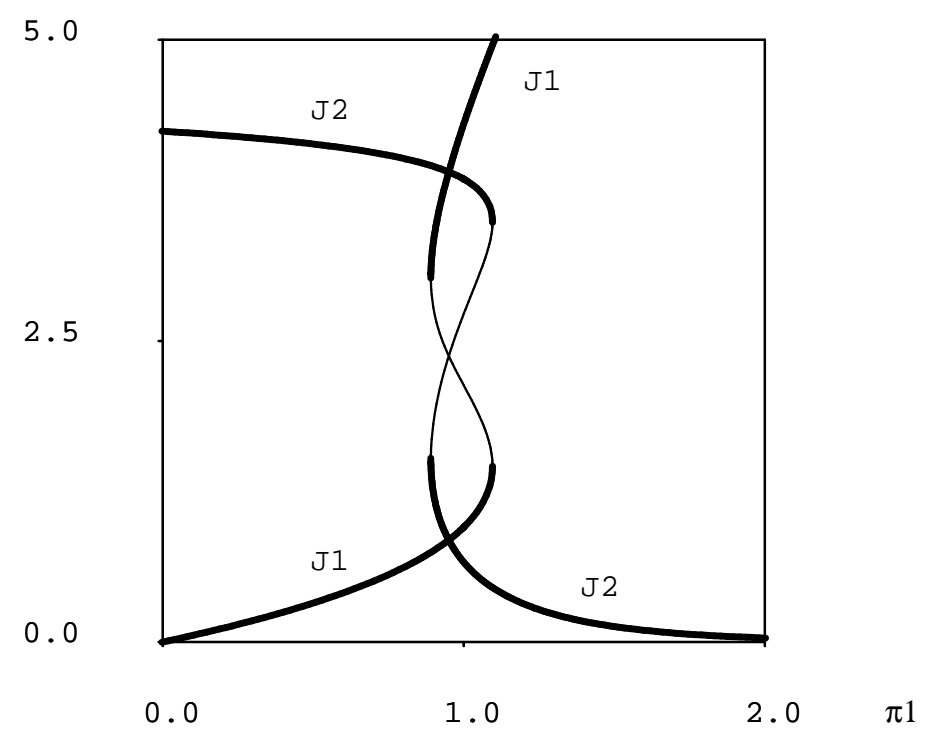

Figure 2: Hysteresis of preference coefficients. Plot of both preference coefficients $J_{1}$ and $J_{2}$ versus $\pi_{1}$, the profit to be obtained from shop number 1 when $\pi_{2}$ the profit to be obtained from shop number 2 is held equal to 1 . $(\beta=0.5$ and $\gamma=0.2)$. The thick lines correspond to stable equilibria for both preference coefficients, $J_{1}$ and $J_{2}$, and the thin lines to unstable equilibria (when $\pi_{1} \simeq \pi_{2}$ ). In the three solutions region, if the initial conditions are such that $J_{1}$ is large (and $J_{2}$ is small), $J_{1}$ remains large when $\pi_{1}$ is decreased, even when $\pi_{1}<\pi_{2}$. The stability of this metastable attractor is lost when $\pi_{1}=0.89$. In a symmetrical manner, the high $J_{2}$ attractor existing at low $\pi_{1}$ can be maintained up to $\pi_{1}=1.095$. (the figure was drawn using GRIND software, De Boer $1983)$.

Figure 2 is represents the preference coefficients $J 1$ and $J 2$ obtained by solving equation (18) by numerical methods (De Boer 1983) for $\pi_{2}=1, \beta=0.5, \gamma=0.2$ and

\footnotetext{
${ }^{15}$ and hence about possible strategies for the sellers.
} 
$\pi_{1}$ varying from 0 to 2 . By following the evolution of preferences on the two branches of figure 2, we can see that buyers can remain loyal to a shop asking for a higher price (which results in a lower profit for the buyer), provided that they became attached to this shop when it asked a lower price. When the most often frequented shop changes its price, the loyalty to that shop describes the upper branch of the loyalty versus profit curve (figure 2). The loyalty remains on the upper branch as long as it exists, i.e. until the point where the slope is vertical. When profit decreases beyond that level, a sudden and discontinuous transition to the lower branch occurs. This is the point when customers change their fidelity and visit the other shop. But, if the first shop reverses its high price/low buyer profit practice when loyalty is on the lower branch, the transition to the higher branch only occurs when the slope of the lower branch becomes vertical, i.e at a higher profit than for the downward transition.

Thus an important qualitative result of the mean field approach is the existence of hysteresis effects: buyers might still have a strong preference for one shop that offered good deals in the past, even though the current deals they offer are less interesting than those now offered by other shops. A consequence of this phenomenon, is that in order to attract customers who are loyal to another shop, a challenger has to offer a profit significantly greater than the profit offered by the well established shop: once preference coefficients have reached equilibrium in the ordered regime, customers switch only for differences in profits corresponding to those where the slopes of the curves $J(\pi)$ in figure 1 are vertical (i.e. not when profits are equalised!). In other words, economic rationality (i.e. choosing the shop offering the best deal) is not ensured in the region where hysteresis occurs.

\section{Results}

\subsection{Indicators of order}

We next proceed to run a number of numerical simulations of our model. This first enables us to check whether the theoretical results obtained from the mean field approximation are consistent with those obtained by running the discrete stochastic process as described by equation (2) and (3). Second, as discussed in the next section, it allows us to compare the simple model with more complicated, analytically intractable versions.

Simulations generate a large number of data about individual transactions such as which shop was visited, purchased quantities, and agents' profits. The organization process itself, involving the dynamics of the buyers' $J_{i j}$ vectors, is harder to monitor. We used two methods to do this.

Firstly, adapting a measure used in Derrida (1986) for instance, we define an order parameter $y_{i}$ by

$$
y_{i}=\frac{\sum_{j} J_{i j}^{2}}{\left(\sum_{j} J_{i j}\right)^{2}},
$$

In the organized regime, when the customer is loyal to only one shop, $y_{i}$ is close to 1 (all $J_{i j}$ except one being close to zero). On the other hand, when a buyer visits $m$ 
shops with equal probability, $y_{i}$ is of order $1 / m$. More generally, $y_{i}$ can be interpreted as the inverse number of shops visited. We usually monitor $y$, the average of $y_{i}$ over all buyers.

Secondly, when the number of shops is small, 2 or 3 , a simplex plot can be used to monitor on-line the loyalty of every single buyer. The first three graphs of figure 3 and 4 display simplex plots of a simulation at different steps. Each agent is represented by a small circle of a specific colour or shade, which represents the agent's probabilistic choice, i.e. the probability distribution over the 3 shops (corresponding to the 3 apices of the triangle). Proximity to one corner is an indication of loyalty to the shop corresponding to that apex. Agents represented by circles close to the center search all shops with equal probability.

\subsection{A simple model}

A simple model was run with 3 sellers and 30 buyers, for a large variety of parameter configurations and initial conditions. In the simulations, time is discrete and buyers receive equal profits when a transaction is made. Sellers' endowments at the beginning of each session are finite, which implies that $\operatorname{Prob}\left(q_{i}>0\right)$ does not have to be one as in the simplest version solved analytically. The figures 3 and 4 correspond to a memory constant $\gamma=0.1$. The critical non-linear parameter corresponding to a unitary profit is then $\beta_{c}=0.3$ (equ. 13). Initial $J_{i j}$ were all 0 . Depending on the value of the non-linear parameter $\beta$, the two predicted dynamic regimes, order and disorder, are observed.

\subsubsection{Disorganized behavior}

For low values of the non-linear parameter $\beta$ buyers never build up any loyalty. This is observed in figure 3 , which describes the dynamics obtained with $\beta=0.15 \beta_{c}$. The daily profit of buyers averaged over all buyers and over 100 days after a transition period of 100 days, is only a fraction ${ }^{16}$ of the buyer's profit per transaction. This is due to all those occasions on which a buyer visited an empty shop. The daily profit of sellers averaged over all sellers and over 100 days after a transition period of 100 days, is only a fraction of ten times the seller profit per transaction (the factor 10 corresponds to the average number of buyers per shop). This difference was also generated indirectly by buyers who visited empty shops since, at the same time some shops with supply were not visited resulting in losses for their owners.

As seen in the simplex plots of figure 3, even at time 50, agents are still scattered around the barycenter of the triangle, an indication for a disordered regime without loyalty of any agent to any shop. Similarly, the order parameter $y$ fluctuates well below 0.50 and thus corresponds to randomly distributed $J_{i j}$. Figure 3 shows that

\footnotetext{
${ }^{16}$ The exact percentage figures depend on the specific demand and supply functions, i.e. on the relationship between purchase and resale price for both, sellers and buyers. The simulations presented here were done with the specific functions discussed in section 5. However, the observed decrease in profit for buyers and sellers is generic.
} 
the performance of shop number 1 exhibits large fluctuations. The same is true for the two other shops.

\subsubsection{Organized behavior}

In sharp contrast, the same analysis performed with $\beta=2 \beta_{c}$ shows a great deal of organisation, see figure 4 .

The average order parameter, $y$, steadily increases to 1 in 200 time steps. As seen on the simplex plot at time 50, each customer has built up loyalty to one shop. Performance of shop number one also stabilizes in time, and variations from stationarity are not observed after 20 time steps.

The daily profit of buyers averaged over all buyers and over 100 days after a transition period of 100 days, is very close to the profit per realised transaction. Because buyers have not changed shops during the last 100 days, sellers learn to purchase the exact quantity needed to satisfy all their buyers and incur no losses as foreseen by the model.

By avoiding daily fluctuations in the number of customers visiting a shop, the ordered regime is beneficial to both customers and sellers, that is both obtain higher profits than in the disorganised situation. In this sense, the ordered regime is Pareto superior to the disordered regime.

\subsubsection{Heterogeneity of buyers and sellers}

\begin{tabular}{|c|c|c|c|c|c|c|c|c|c|}
\hline \multirow[b]{2}{*}{$\beta$} & \multicolumn{7}{|c|}{ histograms of $\mathrm{y}_{i}$} & \multicolumn{2}{|c|}{ order parameters } \\
\hline & 0.3 & 0.4 & 0.5 & 0.6 & 0.7 & 0.8 & 0.9 & $\mathrm{y}$ & $\mathrm{y}_{s}$ \\
\hline 0.3 & 29 & 1 & 0 & 0 & 0 & 0 & 0 & 0.35 & 0.35 \\
\hline 0.6 & 26 & 1 & 2 & 0 & 1 & 0 & 0 & 0.38 & 0.35 \\
\hline 0.9 & 24 & 3 & 1 & 1 & 1 & 0 & 0 & 0.39 & 0.35 \\
\hline 1.2 & 18 & 5 & 2 & 1 & 2 & 1 & 1 & 0.45 & 0.34 \\
\hline 1.5 & 8 & 5 & 2 & 2 & 1 & 6 & 6 & 0.63 & 0.34 \\
\hline 1.8 & 1 & 3 & 5 & 1 & 1 & 7 & 12 & 0.79 & 0.38 \\
\hline 2.1 & 1 & 0 & 0 & 1 & 2 & 6 & 20 & 0.90 & 0.36 \\
\hline 2.4 & 0 & 0 & 0 & 0 & 0 & 6 & 24 & 0.94 & 0.36 \\
\hline 2.7 & 0 & 0 & 0 & 0 & 0 & 1 & 29 & 0.98 & 0.47 \\
\hline
\end{tabular}

Table 1: Histograms and averages of order parameters across the transitions.

In real markets, we expect a mix of buyers with different $\beta$ and $\gamma$ parameters. Thus some buyers will be loyal to certain sellers, while others will continue to search. The same is true for sellers who might offer different prices and thus different profits to buyers. The genericity of the theoretically derived results was tested by numerous simulations.

Table 1 gives a series a results obtained for a uniform distribution of $\gamma_{i}$ coefficients varying from 0.1 to 0.5 among 30 buyers; three shops offer different profits varying 
from -20 perc. to +20 perc. of the middle profit. $\beta_{c}=1.56$ for the median shop $(\gamma=$ $0.03)$ and the median buyer $(\pi=0.057)$. All data were taken after the simulations run for 500 time steps. Different lines of the table correspond to different values of $\beta$ (first column). The next 7 columns represent the histograms of buyers order parameters: the leftmost bin correspond to $0.3<y_{i}<0.4$, the rightmost to $0.9<y_{i}<1.0$. The two rightmost columns are average buyers order parameter $y$ and $y_{s}$ an order parameter for sellers defined by:

$$
y_{s}=\frac{\sum_{j} n_{j}^{2}}{\left(\sum_{j} n_{j}\right)^{2}}
$$

where $n_{j}$ is the number of customers in shop $j$. This series of simulation show that when $\beta$ increases:

- more and more buyers move across the transition towards ordered behaviour, filling the rightmost bins of the histogram; such buyers become loyal to certain sellers, while others continue to search;

- individual transitions remain sharp; buyers maintain either ordered or disordered behavior according to the position of their $\beta_{c}=m \pi / \gamma_{i}$ with respect to $\beta$; note that the intermediate bins have a rather small population with respect to the extremal bins, even for intermediate values of $\beta$;

- Since $y_{s}<0.5$, it is clear that all three shops are visited, even though one is offering a profit 40 perc. above the lowest offered profit.

As also shown in Herreiner (1997), organized or disorganized behavior is here a property of buyers, not a property of markets.

\subsection{Beyond the mean field approximation}

The results of the mean field approach were obtained from a differential equation approximating a discrete time algorithm. They are valid when the changes at each step of the algorithm can be considered small. $\gamma$ thus has to be small, which is true for the simulation results given in figures 2 and 3 .

One of the features noticed by observing on-line the motion of individual buyers in the ordered regime on the simplex plots is that agents sometimes move "backward" towards shops which are not the shops that they "prefer", i.e. those whose with highest preference coefficient. But since for most of the time they move towards preferred shops, these moves only very infrequently make them change shops and preferences. When variables $\gamma$ is increased, these moves have more important consequences.

- Customers might change loyalty from one shop to another one. Increasing $\gamma$ results in larger steps taken by customers on the simplex, which might make them move from one corner to another one in a few time steps. In fact the probability of a given path on the simplex varies as the product of probabilities of individual time steps: if fewer steps are needed the probability that the process will generate such changes becomes higher and they are indeed sometimes observable on-line on the simplex plots. 
- Shops offering higher profits are favored on average by these changes. The stability of fidelity coefficients for shops offering lower profits predicted by the mean field continuous approach becomes metastability in discrete dynamics ${ }^{17}$.

\section{More complicated models and results}

We now briefly discuss some generalisations of the model. Since these more realistic variants are no longer analytically tractable we therefore had to resort to computer simulations to compare their dynamical properties with those of the simple soluble model and with empirical data. Full details about these simulations can be found in Weisbuch et al.(1998). All the variants share the same fundamental mechanism by which buyers choose sellers and the same way of updating preference coefficients as defined in section 2.2.

It is important at this stage to specify the type of comparison that we intend to make between the variants of the model and empirical evidence. We certainly expect some changes to occur at the global level when modifications are introduced in the way in which individual agents make their decisions. Nevertheless, the main point here is to check whether the generic properties of the dynamics are still preserved after these changes. The existence of two distinct, ordered and disordered regimes in which individuals will find themselves, separated by a transition, is such a generic property. On the other hand, we consider as non-generic the values of the parameters at the transition and the values of variables in the ordered or disordered regime. Since even the more elaborate versions of our model are so simplified in comparison with a very complex reality, a direct numerical fit of our model to empirical data would not be very satisfactory, if only because it would involve many parameters which are not directly observable. The search for genericity is based on the conjecture ${ }^{18}$ that the large set of models which share the same generic properties also includes the "true" model of the real system itself.

The search for generic properties frees us from the necessity of having a comprehensive model for all the aspects of the market such as endogenous price dynamics or a fully developed theory of sellers behaviour: as long as various exogenous price dynamics and the different sellers behaviours that we have tested yield the same properties for buyers' behaviour, these properties would also be observed in fully comprehensive models.

For our theoretical discussion it was enough to know the profit realised from a

\footnotetext{
${ }^{17}$ Metastability means that according to initial conditions and probabilistic events, buyers might be attracted towards shops offering lower profit on a short time scale of order $1 / \gamma$, but that shops offering the best deals are selected in the ultra long term (this distinction is similar to the distinction between the long run and the ultra long run introduced in economics by Gale etal (1995). But the times to reach an equilibrium distribution of fidelities can be extremely long depending on $\gamma$ (see Herreiner 1997 for more simulation results). Naturally, in real life, shops losing customers because they offer lower profit have time to readjust and to improve their offers!

${ }^{18}$ This general conjecture, which is basic in the dynamic modeling of complex systems, is proven rigorously for specific systems such as classes of universality in physics (see for instance Pfeuty/Toulouse (1977)) or structural stability in mathematics (see for instance Thom (1975)).
} 
transaction with each firm. A first step towards a more complete model would be to specify explicitly the origin of this profit. For example, one could define the demand faced by the retailers who are the buyers in our model. This in turn would give the optimal price to be set by the seller and the quantity that would be purchased by a buyer. This was done by using a simple hyperbolic specification and assuming that the retailers were local monopolists in order to make the simulations discussed in section 4 .

The one-session model described in section 2 is a considerable simplification of the way buyers search for sellers. As is commonly observed in several markets with the sort of structure we are modeling here, customers that refuse a deal with one seller, usually shop around to find other offers. Indeed this is regarded as the main motivation for refusal in standard search models. An alternative explanation is that customers refuse deals now in order to induce better offers in the future. In either case, to take this into account, we would have to consider a model in which customers are given at least two occasions to purchase goods, with a morning and an afternoon sessions.

This would necessitate specifying the rules by which agents make their decisions taking into account the existence of a second session. Thus sellers would have to decide on the prices to charge in the morning in the light of the transactions they expect to make in the afternoon. They also have to decide on prices in the afternoon given the quantities of goods left on their hands after the morning transactions. Buyers on the other hand, would make a decision as to which prices to accept in the morning given the prices and quantities they expect to be available in the afternoon.

Simulations with an afternoon session do not reveal qualitative changes in the observed behaviour of buyers; we simply observed that when a buyer does not make a transaction in the morning she has a much better chance of making a higher afternoon profit with a new shop that has extra supply. This leads to a more rapid change in the probability of switching to another buyer and to a shorter duration of loyalty.

Another feature that we mentioned previously that would enhance the model is that of the calculation of the amount that sellers wish to supply. To do this optimally would require that the sellers know the probability distribution of the number of customers with which they are faced. This distribution depends on the behaviour of the buyers and is difficult to calculate. However if it is known by the seller, then the optimal quantity to supply is easily computed and is given in Weisbuch et al.(1998).

The last feature of our model which needs to be generalised is that of uniform behaviour by all sellers at any point in time. The ultimate answer to this would be to develop a complete model of sellers' behaviour. However, since our aim in this paper was to explain certain empirical regularities in the behaviour of buyers we confined ourselves to examining the case in which the distribution of prices varied over time. This would suggest that the introduction of strategic pricing behaviour as in the models of Bergemann and Valimaki (1994) and (1996) would not affect the qualitative behaviour of the buyers in our model. 


\section{Empirical Evidence}

In order to see whether there was any empirical evidence of ordered or disordered behaviour of buyers in a market, we started from a data base for transactions on the wholesale fish market in Marseille (M.I.N Saumaty). The data base contains concerns 237162 individual transactions between ca. 1400 buyers and 45 sellers which occurred between 02 - 01 - 1988 to 29 - 06 - 1991. For each transaction the following information is recorded: the name of buyer, the name of seller, the type of fish, the weight of fish, the price, the order in seller's transactions.

The market is organised as in our model, that is, no prices are posted, sellers start with a stock of fish which has to be disposed of rapidly because of its perishable nature. Buyers are either retailers or restaurant owners. Deals are made on a bilateral basis and the market closes at a fixed time. Of course the model is an extreme simplification of the real situation: there are different kinds of fish on the market, each species of fish is heterogeneous, buyers demand different quantities of fish. For a buyer the alternative to purchasing his optimal good is to purchase some inferior alternative.

Direct examination of the data file with the help of standard sorting facilities reveals a lot of organisation in terms of prices and buyer preferences for sellers. In particular, one observes that the most frequent buyers (those who visit the market more than once per week) with very few exceptions visit only one seller, while less frequent buyers visit several sellers, which is consistent with our model. The data will be analysed in this section only in terms of market organisation. Other aspects, such as data classification and price dynamics, which show persistent price dispersion, were analysed in Kirman and Vignes (1991) and Härdle and Kirman (1995).

A first step in comparing our theory with empirical data is to check whether individual buyers display ordered or disordered behaviour during those three and a half years. Since the classical approach to agent behaviour predicts search for the best price, and since searching behaviour implies visiting different shops, any manifestation of order would tend to support our theoretical prediction. If we find evidence of ordered behaviour for certain participants, a second step is then to relate the difference in the observed behaviour of these traders to some difference between their characteristics and those of other buyers.

\begin{tabular}{|l||r|r|r||c|c|}
\hline \multicolumn{1}{|c||}{} & \multicolumn{3}{c||}{$\begin{array}{c}\text { market shares } \\
\text { of }\end{array}$} & \multicolumn{2}{c|}{$\begin{array}{c}\text { monthly purchase } \\
\text { share bought } \\
\text { from one seller }\end{array}$} \\
\cline { 2 - 5 } & largest seller & 2nd & 3rd & $95 \%$ & $80 \%$ \\
\hline \hline cod & $43 \%$ & $14 \%$ & $12 \%$ & $48 \%$ & \\
\hline whiting & $27 \%$ & $8 \%$ & $8 \%$ & $24 \%$ & $53 \%$ \\
\hline sole & $15 \%$ & $14 \%$ & $14 \%$ & $33 \%$ & $55 \%$ \\
\hline
\end{tabular}

Table 2: Loyalty in Cod, Whiting, and Sole Market

For the first step, to check for loyalty of buyers, we consider statistics for cod, 
whiting and sole transactions in $1989^{19}$, see table 2 .

Since we are interested in loyalty issues, we concentrated on the buyers who were present in the market for at least 8 months. As can be seen in the first three columns of table 2, the market for cod is much more concentrated than the market for whiting or sole. In the cod market almost half the buyers (86 of 178) buy more than $95 \%$ of their monthly purchases from one seller only, see the fourth column of table. Also in the whiting and sole market buyers are loyal, but to a lesser degree: more than half of them ${ }^{20}$ buy more than $80 \%$ from one seller. Hence, there are large fractions of loyal buyers in all three markets.

Furthermore, one key conclusion of our model which distinguishes it from a number of other possible explanations of fidelity is that, due to the phase transition, there will be two separate types of behaviour in the market regardless of the underlying distribution of the exogenous variables describing individual characteristics. In other words, even if the exogenous explanatory variables such as frequency of visits or the amounts purchased have an unimodal distribution, the distribution of fidelity will be bimodal.

We then tested the distributions of the dependent variable of our theory, fidelity and of the two exogenous variables frequency of visits and monthly volume of transactions for cod. The histograms are shown in figures 5,6,7. Using a test developed by Scott $(1992)^{21}$ we estimated the probability of different modes. In other words we estimated the probability that a particular value was a local maximum of the density. For fidelity we found that there were two modes with probabilities 0.99 and 0.83 respectively whereas the other two distributions had single modes with 0.99 probabilities. This shows clearly that the two distinct groups of buyers found by examining their fidelity could not be explained by there being two distinct groups of buyers as far as quantities purchased or frequency of visits are concerned.

For the second step, recall that our theory relates loyalty to the parameters $\beta$ (discrimination rate) and $\pi / \gamma$ (cumulated profit). $\beta$, the discrimination parameter is likely to vary from buyer to buyer, but we have no direct way to test it. However, $\pi / \gamma$ is strongly and positively related to monthly purchases of buyers, and we therefore use the latter as a proxy variable. Figure 8 summarises loyalty of buyers in terms of relative frequency of visits to their favorite seller as a function of their monthly purchase of cod on a logarithmic scale. One may observe that loyalty is high in general and that a number of buyers visit only one seller. The fit was done using a non-parametric adaptive smoothing algorithm called LOWESS originally developed by Cleveland in 1979. It is an extension of the usual $\mathrm{k}$ nearest neighbour smoothers since it allows $k$ to vary as a function of the variance in the part of the data being smoothed. It shows shows that loyalty increases with monthly purchase.

All three features are consistent with our theory, and in contradiction with a random search behavior for all buyers.

\footnotetext{
${ }^{19}$ The statistics for other periods of comparable length are very similar.

${ }^{20}$ Whiting 124 of 229 , and sole 154 of 280 .

${ }^{21}$ the test developed by Scott involves the smoothed frequency polygons and is more efficient than tests involving direct smoothing of the histogram itself.
} 


\section{Conclusions}

We have examined a simple model of a market in order to see how the "order" that is observed on many markets for perishable goods develops. "Order" here means the establishment of stable trading relationships over the periods in which the market is open. We focused on the behaviour of buyers to explain how the sharp division into those who are loyal to a particular seller and those who always search could arise. A simple theoretical model yielded this division which is observed on the Marseille fish market. The basic feature of our model is that agents learn by reinforcing those actions, in our case choice of sellers, which proved to be more profitable in the past. Simulations of models which incorporated more general features but which retained the same learning mechanism, showed the same qualitative features as the basic model. Thus what we have shown within the context of an admittedly very simple model is that the presence of "order" and "organisation" in a market is very dependent on, and very sensitive to, the way in which agents react to their previous experience. As has been seen "ordered behaviour" in our model is more efficient in Pareto terms than" disorder" and it is therefore of considerable interest to be able to identify under which conditions such "order" emerges.

\section{Acknowledgment}

We thank Derek Smith for help with the Tk/Tcl interactive display of simulation results, Rob Deboer for the use of his GRIND software, Paul Pezanis and Marlene Mueller for their help in the analysis of Marseille fish market data, Keneth Arrow, Larry Blume, Paul David, Steven Durlauf, Fang Fang Tang, Douglass North, Ariel Rubinstein and Nick Vriend for helpul comments, and Olivier Chenevez, Bernard Derrida, Jean Pierre Nadal and Jean Vannimenus for helpful discussions and important suggestions. The Laboratoire de Physique Statistique of ENS is associated with the CNRS (UA 1306). This work was started during a visit by GW and AK to the Santa Fe Institute (with the help of a NATO Collaborative Research Grant CRG 95 1261) which we thank for its hospitality. 


\section{References}

Anderson S. P., A. de Palma and J.F. Thisse (1993), Discrete Choice Theory of Product Differentiation, Cambridge (MA): MIT Press.

Aoki M. (1996), A New Approach to Macroeconomic Modelling, New York: Cambridge University Press.

Arthur, B.W. and D.A. Lane (1993), "Information Contagion", Structural Changes and Economic Dynamics, 4, 81-104.

Bergemann D. and J. Valimaki (1996), "Market Experimentation and Pricing", Cowles Foundation discussion Paper 1122.

Brock W.A. and S.N. Durlauf (1995), "Discrete Choices with Social Interactions I: Theory", Working Paper 95-10-084, Santa Fe Institute, Santa Fe, NM.

Brout, E. (1965), Phase Transitions, New York (NY): Benjamin.

Cleveland W.S. (1979) "Robust Locally Weighted Regression and Smoothing Scatterplots", Journal of the American Statistical Association, vol. 74, pp.829-836.

De Boer, R. J. (1983), GRIND: Great Integrator Differential Equations, Bioinformatics Group, University of Utrecht, The Nederlands.

Derrida B. (1986) "Phase Transitions in Random Networks of Automata" in: J. Souletie, J. Vannimenus and R. Stora (eds), Chance and Matter, New York (NY): North-Holland.

Diamond P. (1989), "Search Theory", in: J. Eatwell, M. Milgate and P. Newman (eds), The New Palgrave: A Dictionary of Economics, London (UK): Macmillan, 273-79.

Felli, L. and C. Harris (1996),"Learning, Wage Dynamics, and Firm-Specific Human Capital", Journal of Political Economy, 104, 838-868.

Fisher F. M. (1973), " Stability and Competitive Equilibrium in Two Models of Individual Price Adjustment", Journal of Economic Theory, 6, ... .

Föllmer H. (1974) "Random Economies with Many Interacting Agents", Journal of Mathematical Economics, 1/1, 51-62.

Gale J., K. Binmore and L. Samuelson (1995), "Learning to be imperfect: The Ultimatum Game", Games and Economics Behavior, 8, 56-90.

Härdle W. and A. Kirman, (1995), "Non Classical Demand: a Model-Free Examination of Price-Quantity Relation in the Marseilles fish market", Journal of Econometrics, $67, \ldots$.

Herreiner, D. (1997), "Market Organization", mimeo, University of Bonn.

Jackson M. and A.Wolinsky (1996), "A Strategic Model of Social and Economic Networks", Journal of Economic Theory, 71, 44-74.

Kirman, A.P. and A. Vignes (1991), "Price Dispersion. Theoretical Considerations and Empirical Evidence from the Marseilles Fish Market", in K.G. Arrow (ed), Issues in Contemporary Economics, London (UK): Macmillan.

Lesourne, J. (1992), The Economics of Order and Disorder, Oxford: Clarendon Press.

Orléan A. (1995), "Bayesian Interactions and Collective Dynamics of Opinions: Herd Behavior and Mimetic Contagion", Journal of Economic Behavior and Organization, 28, 257-274. 
Pfeuty, P., Toulouse, G. (1977), Introduction to the Renormalization Group and to Critical Phenomena, New York (NY): Wiley.

Scott, D. W. (1992) "Multivariate Density Estimation", John Wiley, New York. jamin.

Thom, R. (1975), Structural Stability and Morphogenesis, New York (NY): Ben-

Weisbuch G. (1990), Complex Systems Dynamics, Redwood City (CA): Addison Wesley.

Weisbuch G., A. Kirman and D. Herreiner (1998), Internal report available on the Web, http://www.lps.ens.fr/ weisbuch/market.ps

\section{A Fix points of $\tanh ()$}

Restate equation (11) as

$$
\tanh (z x)=x, \quad z \in \mathcal{R}, \quad \text { with } \quad x=\frac{\gamma \Delta}{\pi} \quad \text { and } \quad z=\frac{\beta \pi}{2 \gamma}=\frac{\beta}{\beta_{c}},
$$

which is known to have one solution or fix point at $x=0$ if $z \leq 1$; if $z>1$ then there are three solutions.

The stability of these solutions can be analyzed by the slope of $\tanh (z x)$. When $\tanh (z x)>x$ then movement is to the right $(d \Delta / d t>0)$, and conversely:

$$
\begin{aligned}
\tanh (z x)>x & \Longleftrightarrow \pi \cdot \frac{\exp \left(\beta J_{1}\right)-\exp \left(\beta J_{2}\right)}{\exp \left(\beta J_{1}\right)+\exp \left(\beta J_{2}\right)}>\gamma\left(J_{1}-J_{2}\right) \\
& \Longleftrightarrow \pi \frac{\exp \left(\beta J_{1}\right)}{\exp \left(\beta J_{1}\right)+\exp \left(\beta J_{2}\right)}-\gamma J_{1}>\pi \frac{\exp \left(\beta J_{2}\right)}{\exp \left(\beta J_{1}\right)+\exp \left(\beta J_{2}\right)}-\gamma J_{2} \\
& \Longleftrightarrow \frac{d J_{1}}{d t}>\frac{d J_{2}}{d t} \Longleftrightarrow \frac{d \Delta}{d t}>0
\end{aligned}
$$

If $\tanh (z x)$ has one solution, then the slope of $\tanh (z x)$ is flatter than the slope of $x$; the one solution is stable. If $\tanh (z x)$ has three solutions, then at $x=0(\Delta=0)$ the slope of $\tanh (z x)$ is steeper than the slope of $x$, i.e. the central solution is unstable, and then two other solutions are stable.

If $\tanh (z x)$ has three solutions, then the ratio of the preference coefficients at the outer stable solutions is approximately

$$
\frac{J_{1}}{J_{2}}=\exp \left(\frac{\beta \pi}{\gamma}\right),
$$

which can be obtained from equations (9) and (10) if $J_{2} \approx 0$ and $J_{1} \approx \pi / \gamma$.

To determine the speed of transition between the disordered and the ordered regime we calculate the third-order Taylor expansion of $\tanh (z x)$ at $x_{0}=0\left(\Delta_{0}=0\right)$ :

$$
\tanh (0)=0, \quad \tanh ^{\prime}(0)=z, \quad \tanh ^{\prime \prime}(0)=0, \quad \tanh ^{\prime \prime \prime}(0)=-2 z^{3} .
$$


This yields

$$
F\left(x_{0}=0\right)=z x-\frac{(z x)^{3}}{3}=x,
$$

solving for $x(\Delta)$ leads to

$$
x=\sqrt{\frac{3(z-1)}{z^{3}}} \quad \text { and } \quad \Delta=\sqrt{\frac{12\left(\beta-\beta_{c}\right)}{\beta^{3}}}
$$



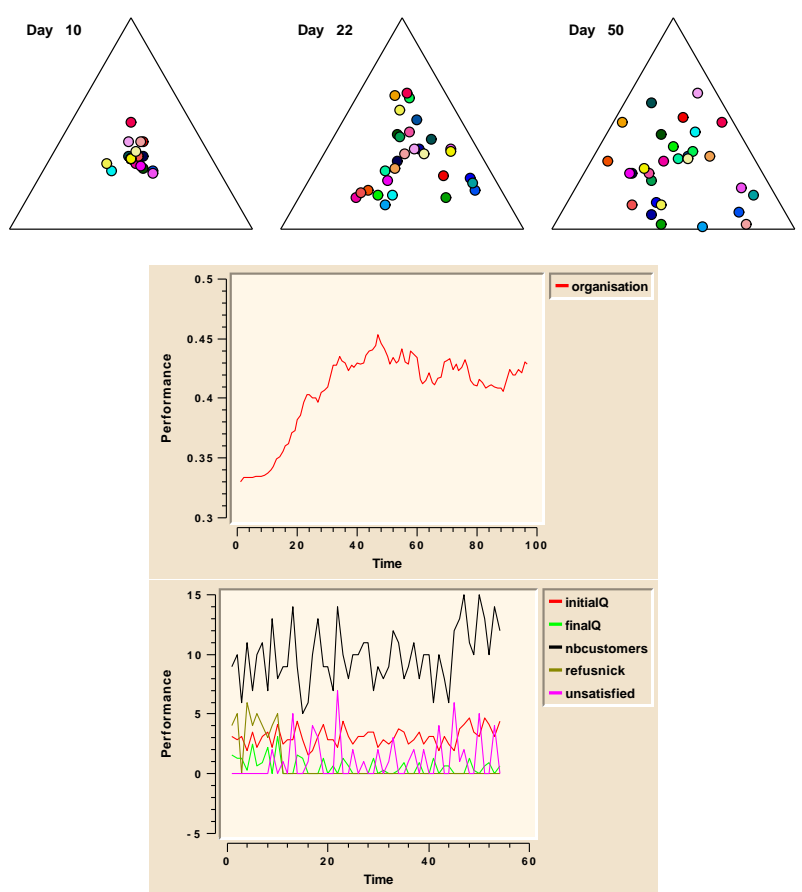

Figure 3: Charts for the disorganized regime. (30 agents visiting 3 shops, with $\gamma=0.1$ and $\beta=0.15 \beta_{c}$ ) The first three graphs monitor market organization by simplex plots taken at time 10,22 and 50. They show that no organization takes place. The fourth graph shows a time plot of the order parameter $y$ (vertical axis: $[0.3,0.5]$ ). The order parameter stays well below 1. The last graph gives a record of shop 1. The time charts display the initial and the final endowment, the number of customers, the number of customers refusing the proposed price (see section 5.2), and the number of unsatisfied customers who did not manage to buy anything. Fluctuations in the market do not decline over time. 

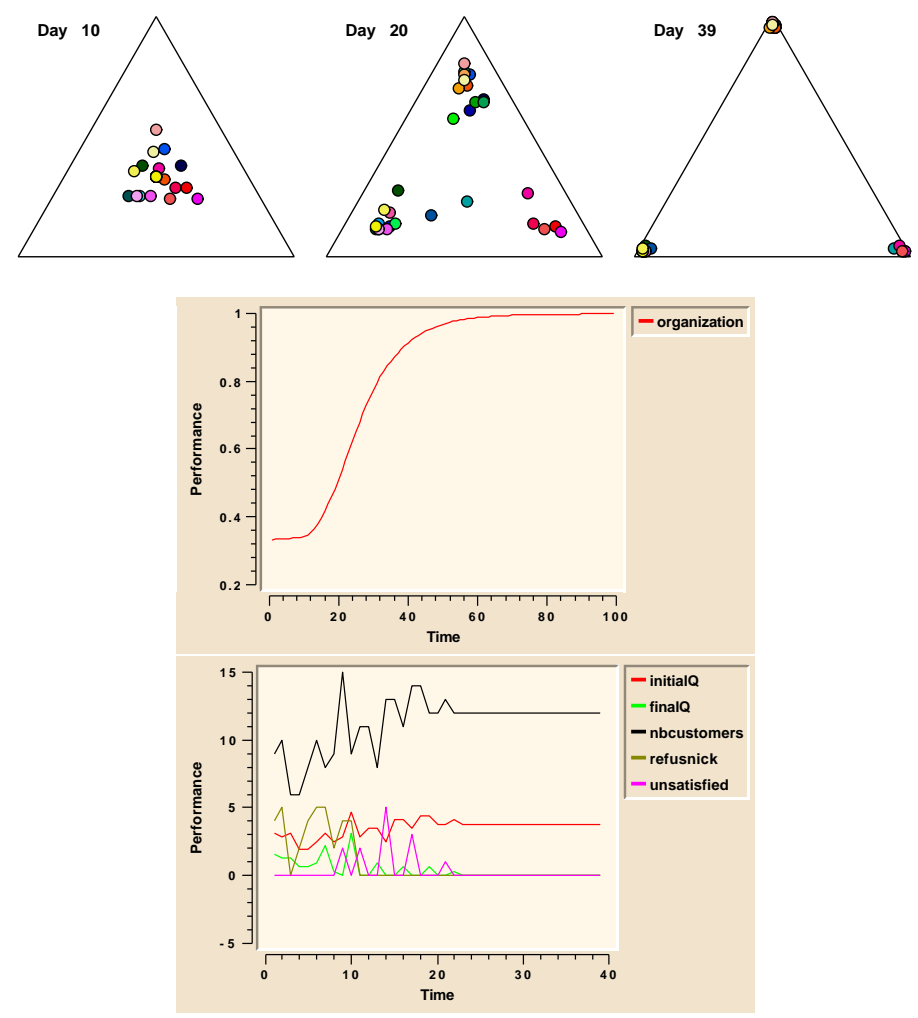

Figure 4: Charts for the organized regime (30 agents visiting 3 shops, with $\gamma=0.1$ and $\beta=2 \beta_{c}$ ). All charts and notation are the same as for figure 3 , except for the scale of the order parameter plot $(y)$. In the three simplex plots, starting from indifference between all three shops, the circles move to the corners representing the preferred shops. Organization takes place. The order parameter $y$ increases steadily from 0.33 to nearly one. The time charts show how fluctuations decrease quickly due to organization. 

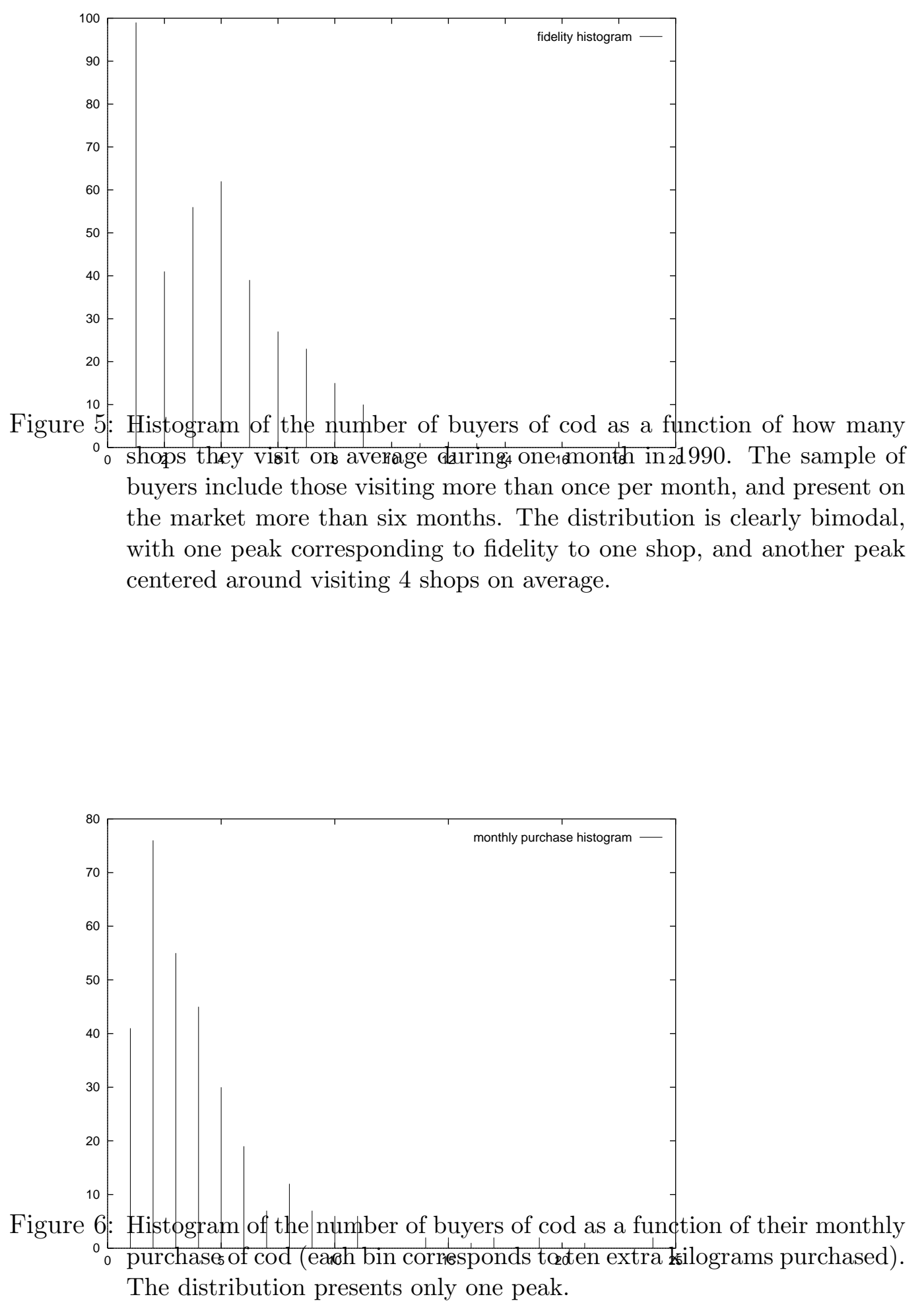


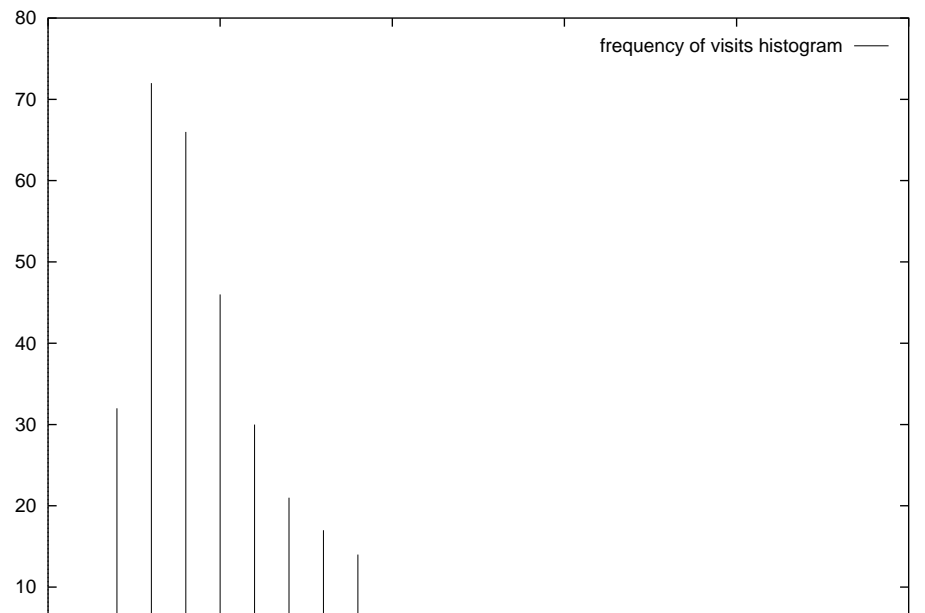

Figure 7: Histogram of the numbler of buyers of cod as a function of their average 0 number of monthlyo visits to thhe fish mærket. Thessdistribution presents only one peak. 


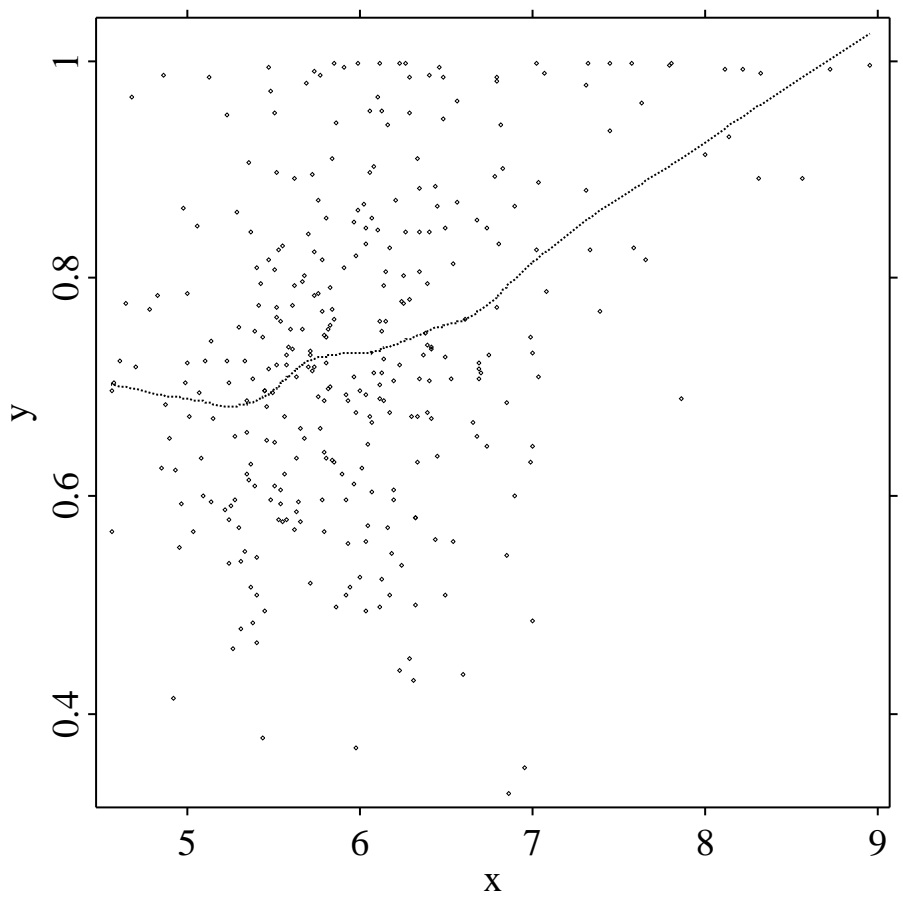

Figure 8: Each dot is an empirical evidence from the Marseilles fish market representing buyer loyalty to his favorite seller (relative frequency of visits) as a function of the logarithm of his monthly purchase of cod in tens of kilograms. Low purchases correspond to infrequent buyers, who generally visit once a week, while large purchase are those of buyers who visit nearly every day the market is open. The fit is a non-parametric adaptive smoothing algorithm which shows that loyalty increases with monthly purchase. 